\title{
Simultaneous bilateral laser therapy accelerates recovery after noise-induced hearing loss in a rat model
}

Jae-Hun Lee ${ }^{1}$, So-Young Chang ${ }^{1}$, Wesley J Moy ${ }^{2}$, Connie Oh ${ }^{2}$, Se-Hyung Kim ${ }^{3}$, Chung-Ku Rhee ${ }^{4}$, Jin-Chul Ahn ${ }^{5}$, Phil-Sang Chung ${ }^{1,4}$, Jae Yun Jung ${ }^{1,4}$, Min Young Lee ${ }^{\text {Corresp. } 4}$

${ }^{1}$ College of Medicine, Dankook University, Beckman Laser Institute Korea, Cheonan, South Korea

2 Beckman Laser Institute and Medical Clinic, University of California, Irvine, California, United States

3 Department of Otolaryngology-Head and Neck Surgery, Jeju National University School of Medicine, Jeju, South Korea

4 Department of Otolaryngology-Head \& Neck Surgery, College of Medicine, Dankook University, Cheonan, South Korea

5 Department of Biomedical Science, College of medicine, Dankook University, Cheonan, South Korea

Corresponding Author: Min Young Lee

Email address: eyeglass210@gmail.com

Noise-induced hearing loss is a common cause of hearing loss. The effects of laser therapy have been investigated from various perspectives, including in wound healing, inflammation reduction, and nerve regeneration, as well as in hearing research. $A$ promising feature of the laser is its capability to penetrate soft tissue; depending on the wavelength, laser energy can penetrate into the deepest part of the body without damaging non-target soft tissues. Based on this idea, we developed bilateral transtympanic laser therapy, which uses simultaneous laser irradiation in both ears, and evaluated the effects of bilateral laser therapy on cochlear damage caused by noise overexposure. Thus, the purpose of this research was to assess the benefits of simultaneous bilateral laser therapy compared with unilateral laser therapy and a control. Eighteen Sprague-Dawley rats were exposed to narrow-band noise at $115 \mathrm{~dB}$ SPL for $6 \mathrm{~h}$. Multiple auditory brainstem responses were measured after each laser irradiation, and cochlear hair cells were counted after the $15^{\text {th }}$ such irradiation. The penetration depth of the $808 \mathrm{~nm}$ laser was also measured after sacrifice. Approximately $5 \%$ of the laser energy reached the contralateral cochlea. Both bilateral and unilateral laser therapy decreased the hearing threshold after noise overstimulation in the rat model. The bilateral laser therapy group showed faster functional recovery at all tested frequencies compared with the unilateral laser therapy group. However, there was no difference in the endpoint ABR results or final hair cell survival, which was analyzed histologically. 
1

\section{Simultaneous Bilateral Laser Therapy Accelerates Recovery} After Noise-Induced Hearing Loss in a Rat Model

Jae-Hun Lee ${ }^{1}$, So-Young Chang ${ }^{1}$, Wesley Moy², Connie $\mathrm{Oh}^{2}$, Se-Hyung Kim³ ${ }^{3}$, Chung-Ku Rhee ${ }^{4}$, Jin-Chul Ahn ${ }^{5}$, Phil-Sang Chung ${ }^{1,4}$, Jae Yun Jung ${ }^{1,4}$, and Min Young Lee ${ }^{4}$

${ }^{1}$ Beckman Laser Institute-Korea, College of Medicine, Dankook University, South Korea

${ }^{2}$ Beckman Laser Institute and Medical Clinic, University of California, Irvine, 1002 Health

Sciences Rd, Irvine CA 92612, United States

${ }^{3}$ Department of Otolaryngology-Head \& Neck Surgery, Jeju National University College of Medicine, Jeju, South Korea

${ }^{4}$ Department of Otolaryngology-Head \& Neck Surgery, College of Medicine, Dankook University, South Korea

${ }^{5}$ Department of Biomedical Science, Dankook University College of Medicine, Cheonan, South Korea

Corresponding Author: Min Young Lee

Department of Otorhinolaryngology-Head \& Neck Surgery, Dankook University Hospital, Seoul, Korea.

E-mail: eyeglass210@gmail.com

Tel: $+82-41-550-1785$

FAX: +82-41-561-3485

Conflict of Interest: None

Running title: Bilateral LLLT on Cochlear 


\section{Abstract}

Noise-induced hearing loss is a common cause of hearing loss. The effects of laser therapy have been investigated from various perspectives, including in wound healing, inflammation reduction, and nerve regeneration, as well as in hearing research. A promising feature of the laser is its capability to penetrate soft tissue; depending on the wavelength, laser energy can penetrate into the deepest part of the body without damaging non-target soft tissues. Based on this idea, we developed bilateral transtympanic laser therapy, which uses simultaneous laser irradiation in both ears, and evaluated the effects of bilateral laser therapy on cochlear damage caused by noise overexposure. Thus, the purpose of this research was to assess the benefits of simultaneous bilateral laser therapy compared with unilateral laser therapy and a control. Eighteen Sprague-Dawley rats were exposed to narrow-band noise at $115 \mathrm{~dB}$ SPL for $6 \mathrm{~h}$. Multiple auditory brainstem responses were measured after each laser irradiation, and cochlear hair cells were counted after the $15^{\text {th }}$ such irradiation. The penetration depth of the $808 \mathrm{~nm}$ laser was also measured after sacrifice. Approximately $5 \%$ of the laser energy reached the contralateral cochlea. Both bilateral and unilateral laser therapy decreased the hearing threshold after noise overstimulation in the rat model. The bilateral laser therapy group showed faster functional recovery at all tested frequencies compared with the unilateral laser therapy group. However, there was no difference in the endpoint ABR results or final hair cell survival,

43 which was analyzed histologically. 


\section{Introduction}

Hearing loss, caused by diverse factors, is an important public health issue. In particular, noise overexposure is considered harmful to hearing function. Intense noise can cause damage to hair cells by increasing oxidative stress, which produces various reactive oxygen species (ROS), such as the superoxide anion $\left(\mathrm{O}_{2}^{-}\right)$(Yamane et al. 1995) and hydrogen peroxide $\left(\mathrm{H}_{2} \mathrm{O}_{2}\right)$ (Ohinata et al. 2000).

Noise exposure can cause a temporary threshold shift (TTS) or a permanent threshold shift (PTS) that will not recover. The type of threshold shift is determined by the intensity and duration of exposure. Several studies with similar levels of noise and exposure times (>100 dB, $>6 \mathrm{~h}$ ) have reported that a PTS occurred after a few minutes or hours of such noise exposure (Buck 1981; Hu et al. 2000; Hu et al. 2006). Both TTS and PTS can occur simultaneously at different frequencies in one cochlea. According to recent research, damage to the auditory neurons, such as at the ribbon synapse and postsynaptic receptors, was found following noise exposure, even after recovery of the hearing threshold (Kujawa \& Liberman 2009).

Laser therapy has been used as a treatment for various symptoms, and its use has been increasing because of its non-invasive nature. After it was approved by the United States Food and Drug Administration, applications of laser therapy have widened in research scope, including wound healing (Anneroth et al. 1988; Grossman et al. 1998; Kana \& Hutschenreiter 1981), inflammation reduction (Boschi et al. 2008; Ferreira et al. 2005), and nerve regeneration (Miloro et al. 2002; Mohammed \& Kaka 2007). Effects of laser therapy have also been reported in the area of hearing research. Some studies have demonstrated significant effects in reducing tinnitus and increasing auditory neuron activation (Littlefield et al. 2010; Medalha et al. 2012; Park et al. 2013). Recently, our group reported a promising recovery effect of laser therapy on cochlear hair cells in an animal study (Rhee et al. 2012). Tamura et al. (2015) also reported a cytoprotective effect of laser therapy in cochlear hair cells against noise overstimulation (Tamura et al. 2015). 
One useful feature of the laser is the capability to penetrate soft tissue; depending on the wavelength, laser energy can penetrate into deep parts of the body without damaging nontargeted soft tissues. This enables the delivery of laser energy from multiple points, which may lead to faster or increased effects of the laser in the target area. In our previous animal experiments, we found improvements in the hearing threshold not only in the laser-irradiated group but also in the contralateral ear (Rhee et al. 2012). This suggests that unilateral laser therapy may affect the contralateral auditory organs. Thus, we measured the degree of laser penetration in the contralateral ear of SD rats and assessed the benefit of simultaneous bilateral laser therapy compared with unilateral laser therapy versus a control group.

\section{Materials and methods}

\section{Animal Subjects}

\section{Acute Acoustic Trauma} centered at $16 \mathrm{kHz}$ with $1 \mathrm{kHz}$ of bandwidth (116 dB SPL). Rats were placed in individual cages to prevent defensive behaviors and these cages were placed in acryl reverberant chambers with a speaker BEYMA CP800Ti (Beyma, Valencia, Spain) attached on top. The traumatic stimulus 
94

95

96

97

was generated with a type 1027 sine random generator (Bruel and Kjaer, Denmark) and amplified with a R300 plus amplifier (inter-Mcorp, Seoul, Korea) for 6 hours. For real time monitoring, a frequency-specific sound level meter (Sound Level Meter - Type 2250, Bruel and Kjaer, Denmark) was used to monitor noise level in the chamber (placed on the floor) every hour so that consistent intensity (116 dB SPL) was maintained during noise exposure.

\section{Auditory Brainstem Response Measurement}

Auditory brainstem responses (ABR) were measured to identify degrees of hearing loss and recovery. The evoked response signal-processing system (System III, Tucker Davis Technologies, Alachua, Florida) was used for ABR measurement. Animals were anesthetized with Zolazepam (Zoletil, Virbac, Carros Cedex, France) and Xylazine (Rompun, Bayer, Leverkusen, Germany) and placed in a sound proof chamber. Three needle electrodes were inserted at vertex (active) and beneath of each pinna (reference and ground), subcutaneously. The tone-burst stimuli $(4,8,12,16$, and $32 \mathrm{kHz})$ were used for experimental measurements and a total 1,024 responses were averaged. Responses were measured in $5 \mathrm{~dB}$ intervals from 90 to $10 \mathrm{~dB}$ SPL and thresholds were determined by the presence of peak within each signal. Hearing thresholds were obtained before and after noise exposure. ABR measurement was also performed during and after laser irradiation (after the $3^{\text {rd }}, 6^{\text {th }}, 9^{\text {th }}, 12^{\text {th }}$, and $15^{\text {th }}$ laser irradiations).

\section{Laser Irradiation Treatment}

An 808nm diode laser (Wontec, South Korea) was used for laser therapy. Each rat in the experimental group was anesthetized and irradiated for $60 \mathrm{mins}\left(165 \mathrm{~mW} / \mathrm{cm}^{2}, 594 \mathrm{~J}\right)$ for 15 days. The density of the laser was calibrated with a laser power meter (FieldMax II-To, Coherent, USA) and detector sensor (Powermax, Coherant, USA). The optical fiber (core fiber $62.5 \mu \mathrm{m}$, cladding $125 \mu \mathrm{m}$ ) was attached to a hollow tube and placed into the external ear canal while leaving a 
117 distance of $1 \mathrm{~mm}$ between the fiber tip and tympanic membrane. Laser irradiation was performed

118 on both the right and left ear simultaneously for the bilateral group and only in the right ear for

119 the unilateral group. The noise only group was anesthetized and the optical fiber was placed

120 into the external ear canal without power. Additional detailed information of the laser is

121 described in Table 1.

122

123

124

125

126

127

128

129

130

131

132

133

134

135

136

137

138

139

Measurement of Laser Energy in the Contralateral Ear

Laser energy was first measured from the contralateral side of the ear using an $808 \mathrm{~nm}$ laser irradiation (calibrated as $165 \mathrm{~mW}$ ) in the SD Rat to confirm the delivery of laser energy to the contralateral cochlea. The rat was sacrificed in $\mathrm{CO}_{2}$ chamber and a secondary decapitation was performed to ensure the rat was no longer alive. The skin and pinna of the test ear (contralateral side from the laser irradiation) were removed to expose the cochlea. The exposed contralateral cochlea was placed above the laser detector and the laser was used to irradiate the ipsilateral external canal with the protocol explained above.

\section{Hair Cell Count}

For the quantitative analysis of outer hair cells (OHCs), whole mounts of the organ of Corti were prepared. Intracardiac perfusion was performed using 4\% Paraformaldehyde (PFA) followed by $0.9 \%$ normal saline. The cochlea was then harvested. After harvesting, the cochlea was fixed in 4\% PFA overnight. After washing with $0.1 \mathrm{M}$ Phosphate-buffered saline (PBS), the cochlea was decalcified with ethylenediaminetetracetic acid (0.5M EDTA, pH 8.0) and was dissected into three parts. The samples were prepared by staining with Phalloidin (PhalloidinFITC, Sigma, USA) and rinsed with 1x PBS. The samples were carefully examined under confocal microscopy (LSM 510 META, Zeiss, Germary) at a magnification of 400X.

\footnotetext{
We chose three representative areas for the quantitative analysis of $\mathrm{OHC}$, which were
} 
140 located at 20,50 , and $80 \%$ from the apex, representing 4, 12, and $32 \mathrm{kHz}$ respectively (Viberg \&

141 Canlon, 2004). Hair cells $<200 \mu m$ in length were counted in each representative area. The

142 morphometric analysis software Image $\mathrm{J}$ (http://rsb.info.nih.goz/ij/) was used to count the

143 number of cells in each section.

\section{Statistical Analysis}

All data were analyzed statistically using the Statistical Package for the Social Sciences software (SPSS, Version 19, IBM, Somers, USA). We performed a Tuckey post hoc test following a Two-way Analysis of Variance (ANOVA) to determine the significance between hearing threshold for ABR measurement and number of hair cells.

\section{Results}

151

152

153

154

155

156

157

158

159

160

161

162

\section{Energy from the $808 \mathrm{~nm}$ Laser was Detected in the Contralateral Ear}

The 808nm laser energy was first measured in the contralateral ear. Using an open air setup between the laser probe and detector, the energy output was determined to be the same at the detector and the displayed output of the laser. $6 \mathrm{~mW}$ of laser energy was measured in the detector at the contralateral ear, while the maximum level of laser energy penetrating the contralateral ear was found to be $8 \mathrm{~mW}$. This result suggests that some laser energy was absorbed prior to exiting the other ear (contralateral ear).

\section{Hearing Loss After Noise Overstimulation}

ABRs were measured before noise exposure to determine the baseline hearing threshold. Mean values (SDs) were 18.61 (5.37), 16.11 (5.57), 16.94 (6.67), 16.11 (5.3), and 16.39 (6.14) 
163 at frequencies of 4, 8, 12, 16, and $32 \mathrm{kHz}$, respectively (Fig. 2). At $24 \mathrm{~h}$ after noise exposure,

164 ABRs were measured again to confirm the degree of hearing loss. Hearing thresholds were

165 increased markedly after noise exposure. Mean values (SD) were 51.11 (6.08), 57.78 (8.44),

16660.28 (6.96), $63.06(4.79)$, and 60.56 (4.82) at frequencies of 4, 8, 12, 16, and $32 \mathrm{kHz}$,

167 respectively (Fig. 2). Thus, these results indicate that overstimulation with a stimulus of $115 \mathrm{~dB}$

168 SPL can cause PTS.

169

Laser Therapy Improved Hearing Recovery in the Bilateral and Unilateral Treated Groups After the sixth laser irradiation, there was a significant difference in the hearing threshold at 16 and $32 \mathrm{kHz}$ between the noise-only and the bilateral laser-treated groups $(p=0.001$ at 16 $\mathrm{kHz}$ and 0.046 at $32 \mathrm{kHz}$; Fig. 2D and E). After the ninth laser irradiation, significant differences existed at all test frequencies between the noise- only and the bilateral laser-treated groups $(p=$ 0.009 at $4 \mathrm{kHz}, 0.04$ at $8 \mathrm{kHz},<0.001$ at $12 \mathrm{kHz}, 0.001$ at $16 \mathrm{kHz}$, and <0.001 at $32 \mathrm{kHz}$ (Fig. 2). The response of the unilateral laser-treated group was significantly different from that of the noise-only group at $32 \mathrm{kHz}$ (Fig. 2E) after the ninth laser irradiation. The difference between the unilateral and the noise-only group increased to $12 \mathrm{kHz}$ and $16 \mathrm{kHz}$ after the twelfth laser irradiation, and the bilateral-treated group showed difference at all frequencies except $8 \mathrm{kHz}$ (Fig. 2C and D). Finally, after the $15^{\text {th }}$ laser irradiation, the hearing threshold at all test frequencies was significant different in the noise-only compared with the bilateral laser-treated group $(p<0.001$ at $4 \mathrm{kHz}, 0.005$ at $8 \mathrm{kHz},<0.001$ at $12 \mathrm{kHz},<0.001$ at $16 \mathrm{kHz}$, and $<0.001$ at $32 \mathrm{kHz}$ ), and the difference between the unilateral group and noise-only group increased to $4 \mathrm{kHz}$ (Fig. 2). This result showed that both bilateral and unilateral laser therapy could reduce the hearing threshold in the SD rat model after noise overstimulation. However, complete recovery of the hearing threshold (to the baseline level) was not achieved. 
189

190

191

192

193

194

195

196

197

198

199

200

201

202

203

204

205

206

207

208

209

210

211

212

213

214

\section{Unilateral Laser Therapy}

A significant difference in the threshold between the bilateral group and the noise-only group was observed from the point of the sixth laser irradiation (at $16 \mathrm{kHz}$ and $32 \mathrm{kHz}$ ) (Fig. 2D and E). In contrast, significant differences between the unilateral group and the noise-only group were observed from the points of the ninth and twelfth laser irradiations (at $32 \mathrm{kHz}$ and $16 \mathrm{kHz}$; Fig. 2D,and E). Furthermore, compared with the hearing threshold recovery in the bilateral group at $4 \mathrm{kHz}, 8 \mathrm{kHz}$, and $12 \mathrm{kHz}$ after the ninth laser irradiation, hearing threshold recovery in the unilateral group at these frequencies (at $4 \mathrm{kHz}$ and $12 \mathrm{kHz}$ ) was observed after the twelfth and $15^{\text {th }}$ laser irradiations (Fig. $2 \mathrm{~A}$ and $\mathrm{C}$ ), respectively. At $8 \mathrm{kHz}$, there was no significant difference between the unilateral group and the noise-only group at any time point. This result indicated that despite the absence of differences in the extent of hearing recovery between the unilateral and bilateral laser therapy groups, the bilateral simultaneous application of laser therapy induced faster (up to 3 days) recovery of the hearing threshold after noise-induced hearing loss compared to the unilateral laser therapy group.

\section{Laser-Treated Group Showed Better Outer Hair Cell (OHC) Preservation in the Basal Turn}

A confocal image of three representative areas is presented in Figure 3. At the apex and the middle area, the averages of OHCs were similar across the three experiment groups (73.67, 72 , and 70.33 at the apex, and $71,72.67$, and 73 at the middle, in the bilateral, unilateral, and noise-only groups, respectively; Fig. 4). However, averages of OHCs at the basal turn were found to be different among each group $(72.67,67.5$, and 59 in the bilateral, unilateral, and noise-only groups, respectively), and both the bilateral and unilateral laser groups showed larger number of OHCs than did the noise-only group $(p=0.0052$ and 0.0006 , respectively; Fig. 4).

\section{Discussion}


Cochlear damage can be variable, and a hearing threshold shift can occur abruptly or progressively, depending on the intensity and duration of noise overstimulation (Clark 1991). In

217 the results of the present study, we found permanent threshold shifts in almost every frequency region examined. These results are consistent with our previous study (Rhee et al. 2012). The results demonstrate that a high level of noise can cause PTS in this rat model. We observed slight improvements in the hearing threshold at low-frequency regions (4 and $8 \mathrm{kHz}$ ) with no treatment, which could be explained as a TTS, because it was not the main target frequency (Clark 1991) of the acoustic overstimulation applied in the current study. Increases in hearing threshold after noise exposure as both PTS and TTS could be a result of loss or dysfunction of $\mathrm{OHC}$ electromotility, which contributes to hearing sensitivity by amplifying the incoming stimulus (Liberman et al. 2002). However, in the present study, we found that the loss of hearing function was not obviously correlated with the histopathology of the OHCs. For this functional loss, we hypothesize that there is some other mechanism of TTS or PTS that may be involved, such as the dispersal of presynaptic ribbons and postsynaptic receptors, which connect the inner hair cells and spiral ganglion (Furman et al. 2013). function similar to our previous study (Rhee et al. 2012). This protection mechanism is considered to be related to the inhibition of iNOS and caspase 3 expression (Tamura et al. 2015), but the details of the underlying mechanism remain unclear. Also, another theory is that this effect may be explained by the balance of free radicals and antioxidants. Before hair cell death, ROS levels increase as a result of noise overexposure. Movement of electrons in hair cells releases energy for converting adenosine diphosphate (ADP) to adenosine triphosphate (ATP) by phosphorylation. During this process, superoxide is generated as an intermediary. When the use of oxygen is increased due to noise exposure, the generation rate of superoxide is also increased by the activity of the mitochondria (Evans \& Halliwell 1999). During noise exposure, mitochondria are strongly stimulated and produce excessive superoxide as a 
241 byproduct. Superoxide can react with other molecules in cochlear hair cells, resulting in

242 subcellular molecular damage. Decreased cochlear blood flow due to noise exposure can also

243 contribute to a deficiency of oxygen in the cochlea. Increased ROS can damage DNA, lipids,

244 and proteins, leading to hair cell death (Evans \& Halliwell 1999).

245 Despite the low penetration level in the contralateral ear, we found faster hearing recovery

246 in the bilateral laser therapy group compared to the unilateral laser therapy group. Additional

247 laser energy may improve the speed of hearing recovery by prompting the endo-organs of the

248 contralateral cochlea. We hypothesize that the laser energy penetrating directly can affect the

249 contralateral cochlea as an activator of cell metabolism. Additionally, the amount of laser energy

250 in the middle of the head, which would be more than that reaching the contralateral cochlea,

251 could have sufficient influence to activate the cochlear nerve or auditory pathway in the midbrain.

252 Moreover, protective actions of cochlear efferent feedback pathway through olivocochlear

253 bundle may also increase recovery. Several studies suggest that the protective effect of the

254 olivocochlear bundle during hearing is allowed to suppress hyper activations of auditory nerve

255 fibers and IHCs (Gifford \& Guinan 1987; Guinan \& Gifford 1988). A damaged olivocochlear

256 bundle by surgical lesion can cause alteration of ABR response, resulting in the increase of hair

257 cell vulnerability related to noise exposure (Maison et al. 2013). Depositing more laser energy

258 into the olivocochelar bundle in the bilateral laser group may increase this protective effect,

259 resulting in a faster recovery compared to unilateral laser group. Additionally, the bilateral laser

260 therapy group did not show improved recovery of the hearing threshold compared to the

261 unilateral laser therapy group after the $15^{\text {th }}$ laser irradiation. This limited effect can be explained

262 by the destruction of the most vulnerable auditory pathways after noise exposure, such as

263 synaptic ribbons (Kujawa \& Liberman 2009). Relatively normal morphologies of OHCs after

264 noise overexposure support this hidden damage theory because the functional loss was much

265 more dramatic compared to the apparently limited hair cell loss found in the histology. There

266 may be additional mechanisms responsible for the functional loss of hearing after noise 
267

268

269

270

271

272

273

274

275

276

277

278

279

280

281

282

283

284

285

286

287

288

289

290

291

292

overexposure, such as synaptic degeneration (Kujawa \& Liberman 2009).

The faster effect of bilateral laser therapy versus unilateral laser therapy is promising for clinical use. Most treatments after hearing loss due to different insults, require early intervention (Ward 1960). There are critical periods of time that can increase the success of a treatment outcome, resulting in a more favorable prognoses (Chen et al. 2007). With bilateral laser therapy, a shorter time frame was required to achieve a desirable outcome; thus, there is a higher chance of staying within the "golden time" for the treatment of hearing loss. Transcanal laser therapy treatments can lead to middle ear complications, such as acute inflammation and perforation of the tympanic membrane (Moon et al. 2016). Applying bilateral laser therapy may reduce the possibility of complications while increasing the effect because laser energy is delivered from two different sites, similar to the protocol for transcranial laser therapy. Multiple site laser irradiation has been used for transcranial laser therapy by several groups (Barrett \& Gonzalez-Lima 2013; Schiffer et al. 2009). These studies reported improvements in cognitive and emotional functions in the brain, with no side effects due to laser irradiation, using lower laser power and irradiating from multiple positions. As such, if estimating the exact location of the cochlea is possible, we may be able to deliver energy to the cochlea from multiple sites transcranially. However, no methodology for transcranial aiming toward the cochlea has yet to be established or developed.

To apply bilateral laser therapy in the clinic, some practical issues must be considered.

Because of anatomical differences between humans and rodents, the effects of laser energy on the contralateral side would be different in humans compared to rodents. The larger distance from one ear to the other may limit the delivery of laser energy; however, the beneficial effect of bilateral laser therapy would be expected to remain if the mechanism involves targeting the brainstem. Increasing the power of the laser may be another approach to deliver energy to the other ear, but this could cause unwanted side effects, resulting in local burning and tympanic perforation. Thus, increasing the power of transcanal laser irradiation should be carefully 
293 considered before translation to clinical application.

295 Conclusions

296 The present study showed positive effects of bilateral laser therapy after noise-induced hearing

297 loss in an animal model. The results suggest that the use of bilateral laser therapy in a clinical

298 setting may improve the therapeutic effects on hearing while minimizing side effects. 
301

302

303

304

305

306

307

308

309

310

311

312

313

314

315

316

317

318

319

320

321

322

323

324

325

326

327

328

329

330

331

332

333

334

335

336

Anneroth G, Hall G, Ryden H, and Zetterqvist L. 1988. The effect of low-energy infra-red laser radiation on wound healing in rats. British Journal of Oral and Maxillofacial Surgery 26:12-17. Barrett D, and Gonzalez-Lima F. 2013. Transcranial infrared laser stimulation produces beneficial cognitive and emotional effects in humans. Neuroscience 230:13-23.

Boschi ES, Leite CE, Saciura VC, Caberlon E, Lunardelli A, Bitencourt S, Melo DA, and Oliveira JR. 2008. Anti-inflammatory effects of low-level laser therapy $(660 \mathrm{~nm})$ in the early phase in carrageenan-induced pleurisy in rat. Lasers in surgery and medicine 40:500-508.

Buck K. 1981. Influence of different presentation patterns of a given noise dose on hearing in guineapig. Scandinavian audiology Supplementum 16:83-87.

Chen C-J, Dai Y-T, Sun Y-M, Lin Y-C, and Juang Y-J. 2007. Evaluation of auditory fatigue in combined noise, heat and workload exposure. Industrial health 45:527-534.

Clark WW. 1991. Recent studies of temporary threshold shift (TTS) and permanent threshold shift (PTS) in animals. The Journal of the Acoustical Society of America 90:155-163.

Evans $\mathrm{P}$, and Halliwell B. 1999. Free radicals and hearing: cause, consequence, and criteria. Annals of the New York Academy of Sciences 884:19-40.

Ferreira D, Zangaro R, Villaverde AB, Cury Y, Frigo L, Picolo G, Longo I, and Barbosa D. 2005.

Analgesic effect of He-Ne $(632.8 \mathrm{~nm})$ low-level laser therapy on acute inflammatory pain.

Photomedicine and laser surgery 23:177-181.

Furman AC, Kujawa SG, and Liberman MC. 2013. Noise-induced cochlear neuropathy is selective for fibers with low spontaneous rates. Journal of neurophysiology 110:577-586.

Grossman N, Schneid N, Reuveni H, Halevy S, and Lubart R. 1998. 780 nm low power diode laser irradiation stimulates proliferation of keratinocyte cultures: involvement of reactive oxygen species. Lasers in surgery and medicine 22:212-218.

Hu BH, Guo W, Wang PY, Henderson D, and Jiang SC. 2000. Intense noise-induced apoptosis in hair cells of guinea pig cochleae. Acta oto-laryngologica 120:19-24.

$\mathrm{Hu}$ BH, Henderson D, and Nicotera TM. 2006. Extremely rapid induction of outer hair cell apoptosis in the chinchilla cochlea following exposure to impulse noise. Hearing research 211:16-25.

Kana JS, and Hutschenreiter G. 1981. Effect of low-power density laser radiation on healing of open skin wounds in rats. Archives of Surgery 116:293-296.

Kujawa SG, and Liberman MC. 2009. Adding insult to injury: cochlear nerve degeneration after "temporary" noise-induced hearing loss. The Journal of Neuroscience 29:14077-14085.

Liberman MC, Gao J, He DZ, Wu X, Jia S, and Zuo J. 2002. Prestin is required for electromotility of the outer hair cell and for the cochlear amplifier. Nature 419:300-304.

Littlefield PD, Vujanovic I, Mundi J, Matic AI, and Richter CP. 2010. Laser stimulation of single auditory nerve fibers. The Laryngoscope 120:2071-2082. 
337

338

339

340

341

342

343

344

345

346

347

348

349

350

351

352

353

354

355

356

357

358

359

360

361

362

363

364

365

366

367

368

369

370

371

372

Medalha CC, Di Gangi GC, Barbosa CB, Fernandes M, Aguiar O, Faloppa F, Leite VM, and Renno ACM. 2012. Low-level laser therapy improves repair following complete resection of the sciatic nerve in rats. Lasers in medical science 27:629-635.

Miloro M, Halkias LE, Mallery S, Travers S, and Rashid RG. 2002. Low-level laser effect on neural regeneration in Gore-Tex tubes. Oral Surgery, Oral Medicine, Oral Pathology, Oral Radiology, and Endodontology 93:27-34.

Mohammed IF, and Kaka LN. 2007. Promotion of regenerative processes in injured peripheral nerve induced by low-level laser therapy. Photomedicine and laser surgery 25:107-111.

Moon T-H, Lee MY, Jung JY, Ahn J-C, Chang S-Y, Chung P-S, Rhee C-K, Kim Y-H, and Suh M-W. 2016. Safety assessment of trans-tympanic photobiomodulation. Lasers in medical science:1-11.

Ohinata Y, Miller JM, Altschuler RA, and Schacht J. 2000. Intense noise induces formation of vasoactive lipid peroxidation products in the cochlea. Brain research 878:163-173.

Park YM, Na WS, Park IY, Suh M-W, Rhee C-K, Chung P-S, and Jung JY. 2013. Trans-canal laser irradiation reduces tinnitus perception of salicylate treated rat. Neuroscience letters 544:131-135. Rhee C-K, Bahk CW, Kim SH, Ahn J-C, Jung JY, Chung P-S, and Suh M-W. 2012a. Effect of low-level laser treatment on cochlea hair-cell recovery after acute acoustic trauma. Journal of biomedical optics 17:0680021-0680026.

Schiffer F, Johnston AL, Ravichandran C, Polcari A, Teicher MH, Webb RH, and Hamblin MR. 2009.

Psychological benefits 2 and 4 weeks after a single treatment with near infrared light to the forehead: a pilot study of 10 patients with major depression and anxiety. Behavioral and Brain Functions 5:1. Tamura A, Matsunobu T, Mizutari K, Niwa K, Kurioka T, Kawauchi S, Satoh S, Hiroi S, Satoh Y, and Nibuya M. 2015. Low-level laser therapy for prevention of noise-induced hearing loss in rats. Neuroscience letters 595:81-86.

Ward WD. 1960. Recovery from high values of temporary threshold shift. The Journal of the Acoustical Society of America 32:497-500.

Yamane H, Nakai Y, Takayama M, Konishi K, Iguchi H, Nakagawa T, Shibata S, Kato A, Sunami K, and Kawakatsu C. 1995. The emergence of free radicals after acoustic trauma and strial blood flow. Acta oto-laryngologica 115:87-92.

Gifford ML, and Guinan JJ. 1987. Effects of electrical stimulation of medial olivocochlear neurons on ipsilateral and contralateral cochlear responses. Hearing research 29:179-194.

Guinan JJ, and Gifford ML. 1988. Effects of electrical stimulation of efferent olivocochlear neurons on cat auditory-nerve fibers. I. Rate-level functions. Hearing research 33:97-113.

Maison SF, Usubuchi H, and Liberman MC. 2013. Efferent feedback minimizes cochlear neuropathy from moderate noise exposure. The Journal of Neuroscience 33:5542-5552. 

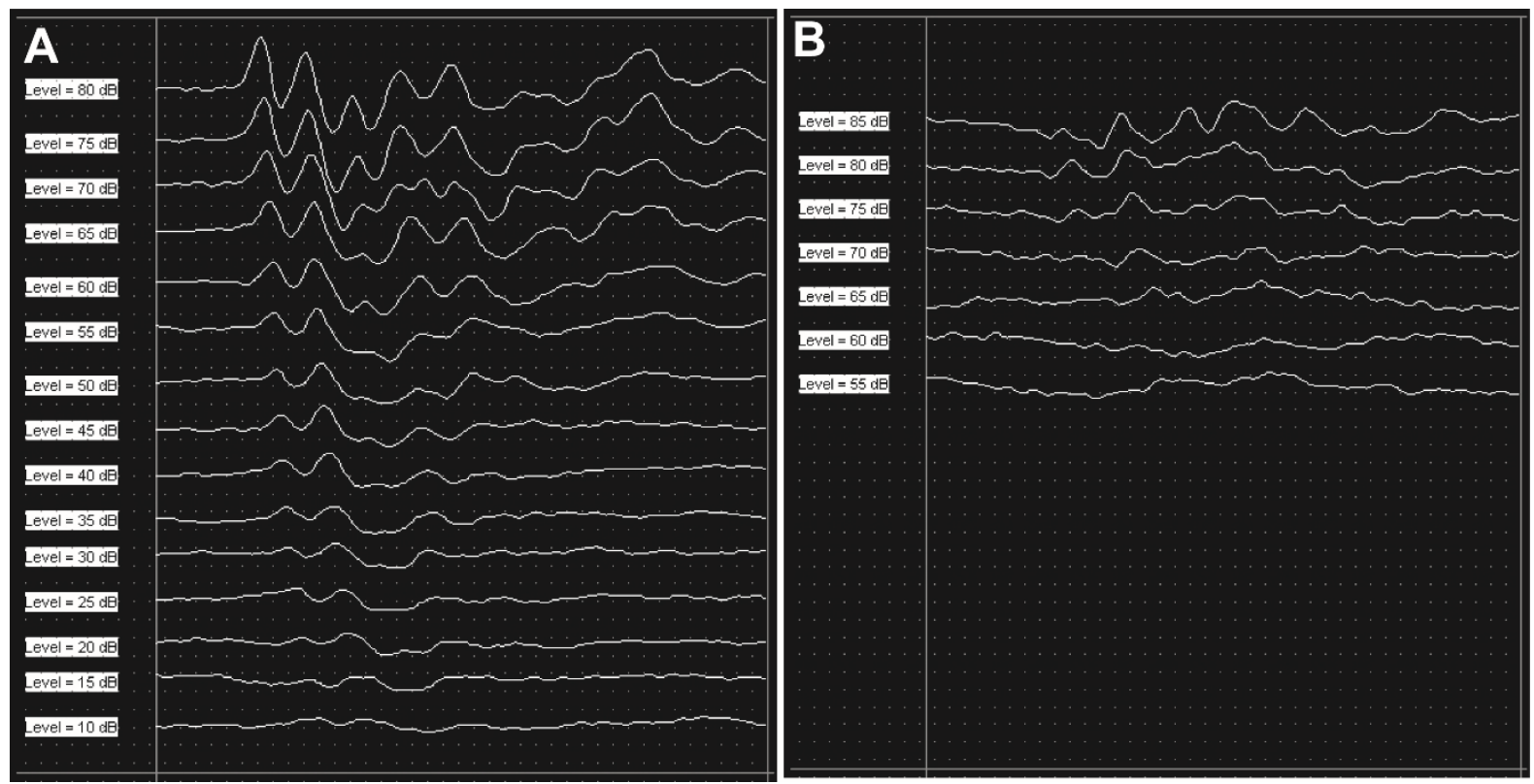

Figure 1. Results of ABR measurement. Consistent peaks were recorded at $16 \mathrm{kHz}$ during $\mathrm{ABR}$ measurement as a baseline (A). After six hours of noise exposure, the overall amplitude of the peaks were reduced compared to the baseline result, and the peaks disappeared under $65 \mathrm{~dB}$ SPL at the same test frequency $(B)$. 


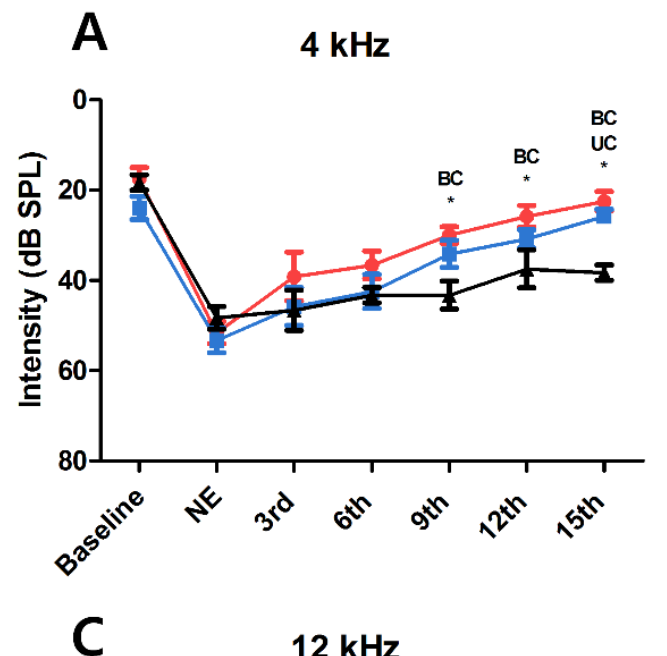

B $\quad 8 \mathrm{kHz}$
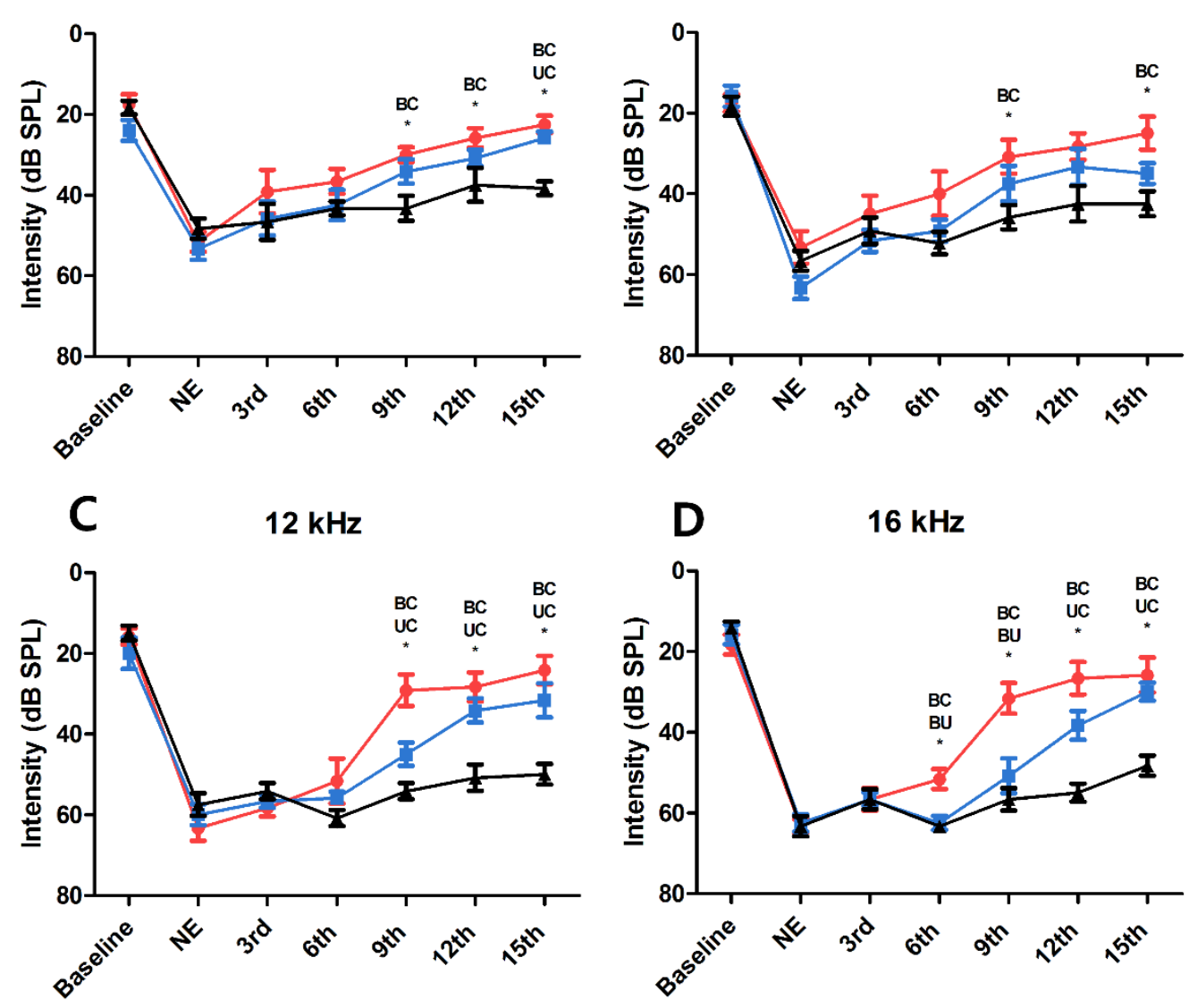

390

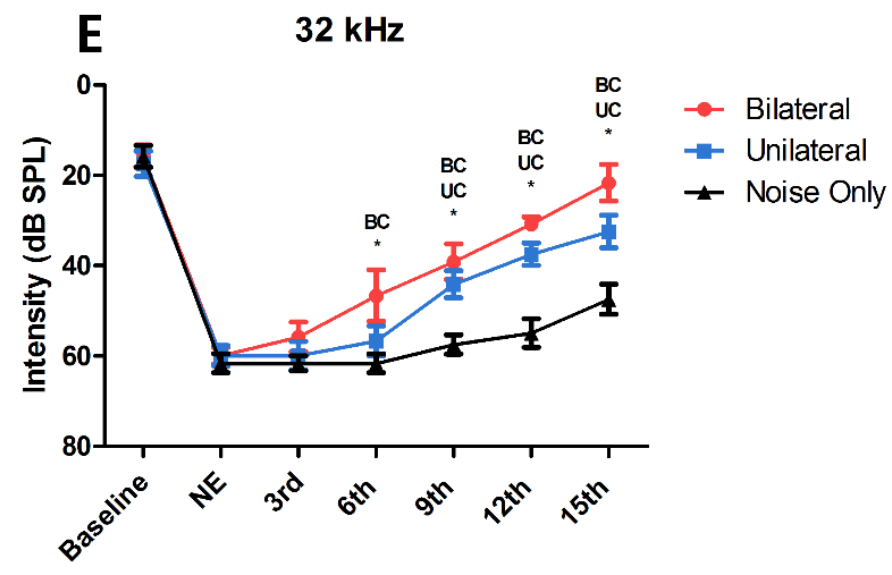

391 Figure 2. Changes in hearing threshold at each ABR measurement. At every tested frequency,

392 the result of the bilateral LLLT group showed faster hearing recovery than the unilateral LLLT

393 group, asterisk represents the statistical difference between the two groups noted above itself

394 (NE: noise exposure, BC: Bilateral and Control, BU: Bilateral and Unilateral, UC: Unilateral and 
395 Control). 

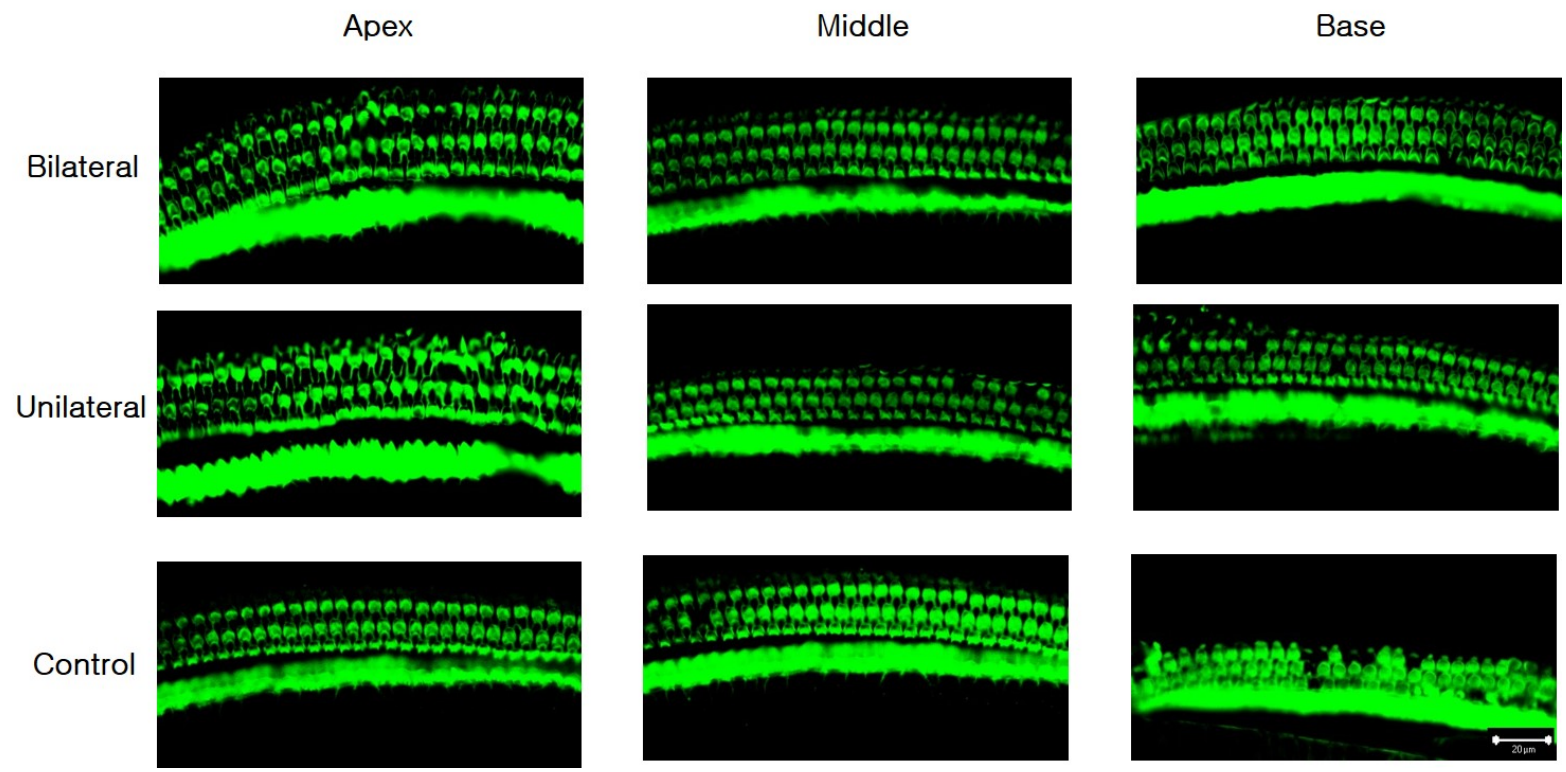

Figure 3. Representative confocal images of hair cells at three different locations (apex, middle, the cochlea in the noise-only group.

400

401 


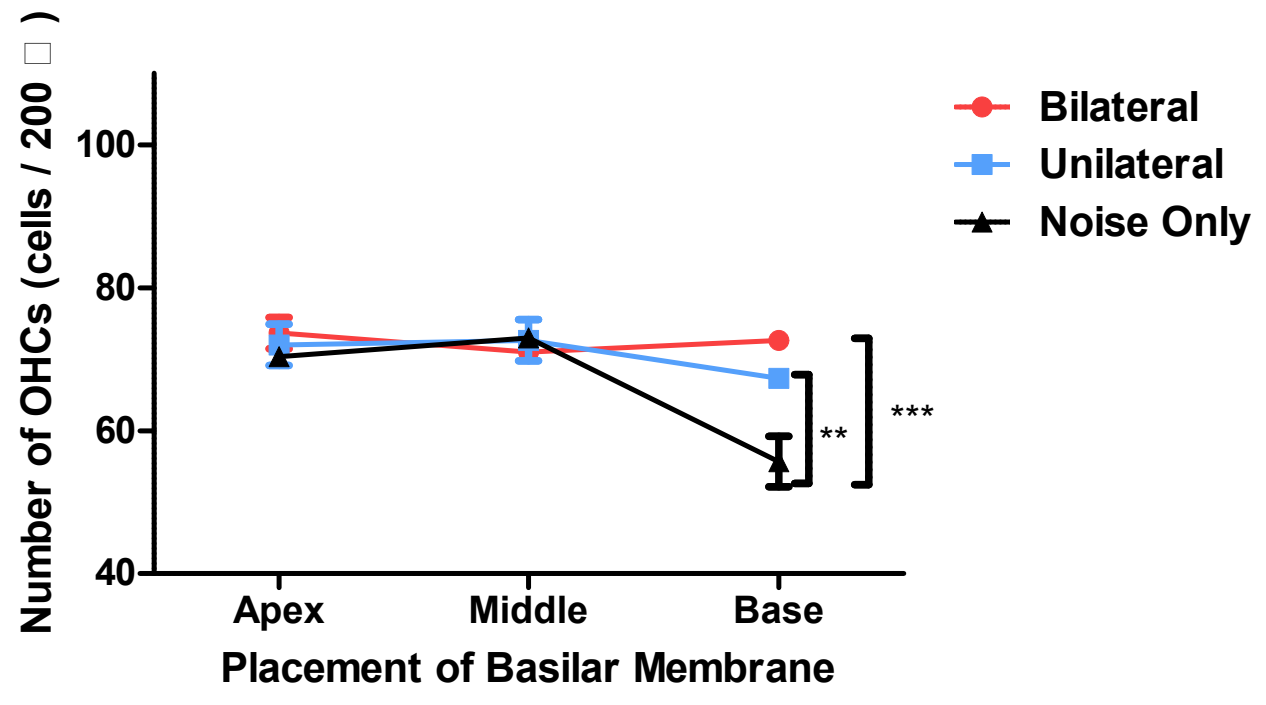

402

403 Figure 4. Numbers of OHCs in three parts of the basilar membrane in each group. The bilateral 404 and unilateral laser groups showed significantly larger numbers of OHCs at the base part of the 405 basilar membrane $\left({ }^{* *} p<0.01,{ }^{* * *} p<0.001\right)$.

406 
407 Table 1. Laser (Photobiomodulation) parameter

\begin{tabular}{|c|c|}
\hline Parameter & Laser group (Bilateral and Unilateral) \\
\hline Power (mW) & 185 \\
\hline $\begin{array}{l}\text { Beam spot size at target } \\
\qquad(\mathrm{cm} 2)\end{array}$ & 0.22 \\
\hline $\begin{array}{c}\text { Irradiance at target } \\
(\mathrm{mW} / \mathrm{cm} 2) \text { power density }\end{array}$ & 841 \\
\hline Exposure duration (s) & 3,600 \\
\hline $\begin{array}{l}\text { Radiant exposure }(\mathrm{J} / \mathrm{cm} 2) \\
\text { fluence }\end{array}$ & 2,700 \\
\hline Radiant energy $(\mathrm{J})$ & 594 \\
\hline Number of points irradiated & 1 \\
\hline Area irradiated (cm2) & 0.22 \\
\hline Application technique & Through tympanic membrane \\
\hline $\begin{array}{c}\text { Number and frequency of } \\
\text { treatment sessions }\end{array}$ & Once a day for 15 days \\
\hline Total radiant energy $(\mathrm{J})$ & 8,910 \\
\hline
\end{tabular}

408

409 
410 The English in this document has been checked by at least two professional editors, both native 411 speakers of English. For a certificate, please see:

412

413 http://www.textcheck.com/certificate/4yrTr7 